\title{
Curve Tracking Control for Autonomous Vehicles with Rigidly Mounted Range Sensors
}

\author{
Jonghoek Kim, Fumin Zhang, and Magnus Egerstedt
}

\begin{abstract}
In this paper, we present a feedback control law to make an autonomous vehicle with rigidly mounted range sensors track a desired curve. In particular, we consider a vehicle which has two range sensors that emit rays perpendicular to the velocity of the vehicle. Under such a sensor configuration, singularities are bound to occur in the feedback control law. Thus, to overcome this, we derive a hybrid strategy of switching between control laws close to the singularity.
\end{abstract}

\section{INTRODUCTION}

Curve tracking control is fundamental for autonomous vehicles following desired paths, e.g. staying in lanes, or avoiding obstacles. An example in which this becomes relevant is when an autonomous vehicle is to follow the curb or the lane markings of a car. In fact, Fig.1 shows the mobile vehicle that represented Georgia Tech in the DARPA Urban Grand Challenge in 2007. As one of this vehicle's lane perception strategies, two rigidly mounted range sensors(lidars) were installed on both sides of the vehicle. At each instant of time, the vehicle emits a ray forming a fixed angle with the velocity of the vehicle. When the sensor ray intersects a lane, it detects a point on the lane. From the distance measurements taken, the autonomous vehicle estimates the curvature of the lane at the detected point, the distance from the point, and the angle between the heading direction of the vehicle and the tangent vector to the lane.

In this paper, we design a curve tracking control law that uses this information to produce the desired lane following behavior as a component in the Georgia Tech Urban Grand Challenge system. It should be noted that our results can be applied to a number of other types of autonomous vehicles with rigidly mounted range sensors.

The literature is abundant with papers on trajectory tracking for autonomous vehicles. For example, in [1], a reference point on the contour being tracked moves along the reference trajectory while the vehicle follows it. Otherwise, the reference point might stop to wait for the vehicle. In [2] and [3], a gyroscopic feedback

Jonghoek Kim, Fumin Zhang, and Magnus Egerstedt are with the School of Electrical and Computer Engineering, Georgia Institute of Technology, USA. Email: jkim37@mail.gatech.edu, \{fumin, magnus\}@ece.gatech.edu law was used to model the interaction of a particle with an image particle representing the closest point on a closed curve bounding an obstacle. This controller design method was generalized to cooperative motion patterns on closed curves for multiple vehicles in [4], [5], [6]. The closest point is also used for path following in [7]. In [8], sensors provide measurements at different fixed points in front of the vehicle. From the data, a recursive spline is updated, then tracked by applying a suitable feedback control law. Similarly, the problem of tracking a ground curve is formulated as controlling the shape of the curve in the image plane in [9]. Other vision-based path following methods can be found in [10], [11], [12]. To achieve motion camouflage, biologically plausible feedback law which has an intuitive physical interpretation is shown in [13]. The authors of [14] determined bound of the tolerance sampling interval so that the vehicle stays in the lane. A feedback linearization approach and Lyapunov-oriented control designs are presented to make a mobile vehicle converge to a predefined path [15]. Curve tracking for atomic force microscope is considered in [16]. A decentralized coordination algorithm for multiple vehicles to locate and track a dynamic perimeter is presented in [17]. The authors of [18] proposed a control law for following isolines in a potential field not relying on higher order characteristics of the field such as the gradient at a point or the curvature of the isolines.

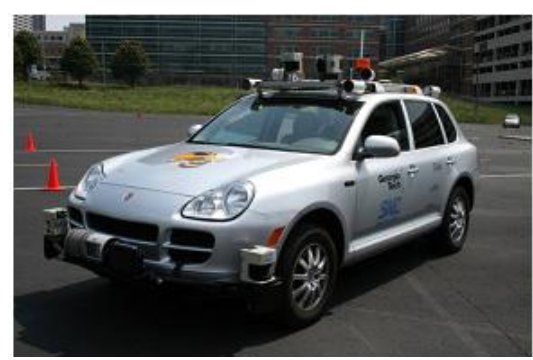

Fig. 1. The Sting-1 vehicle during testing at Georgia Tech.

Conditions for nonlinear switched system to be asymptotically stable were presented in [19]. In [20],[21], and [22], multiple Lyapunov functions were presented for making a switched system to be Lyapunov 
stable. Furthermore, three composite quadratic Lyapunov functions are used for the construction of stabilizing laws for discrete-time switched systems [23]. In [24], the authors proposed control laws that switch between an approximate control law when the system is near a singularity, and an exact control law when the system is bound away from the singularity.

A similar philosophy is pursued in this paper. In fact, the control law for boundary following presented in this paper has two attractive features. First, to overcome singularities of a Lyapunov function-oriented control law, a switched controller is presented to make the system asymptotically stable. Second, our approach allows a vehicle to follow a boundary curve using only two range sensors. Hence, this approach does not require wide-angle scanning that is commonly assumed by other boundary-following algorithms.

\section{BOUNDARY-Following MODEL WITH RIGIDLY MOUNTED RANGE SENSORS}

Consider a vehicle with two range sensors that emit rays forming a fixed angle $\alpha$ with the velocity of the vehicle. When a boundary curve is presented in the plane, the sensor ray will intersect the boundary and detect a point $\vec{r}_{2}$, which will be called the detected point. Here, $\vec{r}_{1}$ is the position of the vehicle. Hence, the relative position between the vehicle and the detected point is $\vec{r}_{\alpha}=\vec{r}_{2}-\vec{r}_{1}$, and $\phi$ is the angle measured counterclockwise from the tangent vector $\vec{x}_{2}$ at the detected point to the heading direction of the vehicle $\vec{x}_{1}$.

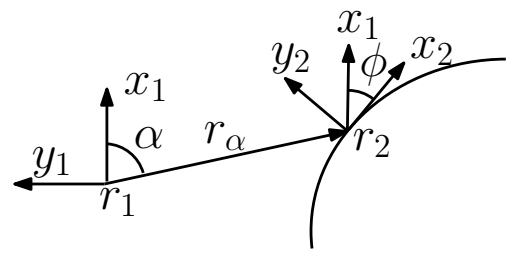

Fig. 2. A vehicle with a rigidly mounted sensor at angle $\alpha$ and a boundary curve in its environment.

We first establish two Frenet-Serret frames [25]: one at the vehicle, the other at the detected point, as shown in Fig. 2. These two frames satisfy the Frenet-Serret equations:

$$
\begin{aligned}
& \dot{\vec{r}}_{1}=v_{1} \vec{x}_{1} \\
& \dot{\vec{x}}_{1}=v_{1} u \vec{y}_{1} \\
& \dot{\vec{y}}_{1}=-v_{1} u \vec{x}_{1}
\end{aligned}
$$

$$
\begin{aligned}
& \dot{\vec{r}}_{2}=\dot{s} \vec{x}_{2} \\
& \dot{\vec{x}}_{2}=\dot{s} \kappa \vec{y}_{2} \\
& \dot{\vec{y}}_{2}=-\dot{s} \kappa \vec{x}_{2},
\end{aligned}
$$

where $v_{1}$ is the speed control, $\kappa$ is the curvature of the curve at the detected point, and $s$ is the arc-length parameter of the curve. Here, $u$ is the steering (i.e., curvature) control we apply to avoid colliding with the obstacle and to achieve boundary following.

We may choose the positive direction of the boundary curve such that

$$
\vec{x}_{1} \cdot \vec{x}_{2}=\cos (\phi)>0 \text {. }
$$

When the curve is convex, i.e., curving away from the vehicle, we have $\kappa<0$. When the curve is concave, i.e. curving towards the vehicle, the curvature $\kappa>0$. The above settings for the interaction between the vehicle and the boundary curve were introduced in [2].

The key idea of curve tracking control is to control the relative motion between the vehicle and the detected point. For this purpose, we develop a set of equations that govern the relative motion.

The relative position between the free particle and the detected point is $\left(\vec{r}_{\alpha}=\vec{r}_{2}-\vec{r}_{1}\right)$. In Fig. 2, $\alpha$ is defined as the angle formed by $\vec{r}_{\alpha}$ and $\vec{x}_{1}$. Also, let $r_{\alpha}=\left\|\vec{r}_{\alpha}\right\|$. Then

$$
\vec{r}_{\alpha} \cdot \vec{x}_{1}=\cos (\alpha) r_{\alpha}
$$

To derive the relative motion equations, we need to find $\dot{r}_{\alpha}, \dot{s}$ and $\dot{\phi}$.

We first obtain an equation between $\dot{r}_{\alpha}$ and $\dot{s}$. We take the time derivative of $\vec{r}_{\alpha}$ using (1) and (2) to get

$$
\dot{\vec{r}}_{\alpha}=\dot{s} \vec{x}_{2}-v_{1} \vec{x}_{1} \text {. }
$$

Differentiating (4) with respect to time on both sides, we obtain

$$
\dot{\vec{r}}_{\alpha} \cdot \vec{x}_{1}+\vec{r}_{\alpha} \cdot \dot{\vec{x}}_{1}=\cos (\alpha) \dot{r}_{\alpha}
$$

And then, replacing $\dot{\vec{x}}_{1}$ by $v_{1} u \vec{y}_{1}$, we get

$$
\dot{\vec{r}}_{\alpha} \cdot \vec{x}_{1}+\vec{r}_{\alpha} \cdot v_{1} u \vec{y}_{1}=\cos (\alpha) \dot{r}_{\alpha} .
$$

Replacing $\dot{\vec{r}}_{\alpha}$ in (7) by (5), we obtain

$$
\left(\dot{s} \vec{x}_{2}-v_{1} \vec{x}_{1}\right) \cdot \vec{x}_{1}+\vec{r}_{\alpha} \cdot v_{1} u \vec{y}_{1}=\cos (\alpha) \dot{r}_{\alpha} .
$$

We observe that, in Fig. 2, the angle formed by $\vec{x}_{1}$ and $\vec{x}_{2}$ is $\phi$. Also, the angle formed by $\vec{r}_{\alpha}$ and $\vec{y}_{1}$ is $\left(\frac{\pi}{2}+\alpha\right)$. Therefore, we get

$$
\dot{s} \cos (\phi)=v_{1}\left(1+\sin (\alpha) r_{\alpha} u\right)+\cos (\alpha) \dot{r}_{\alpha}
$$


Note that $r_{\alpha}^{2}=\left\|\vec{r}_{\alpha}\right\|^{2}=\left(\vec{r}_{2}-\vec{r}_{1}\right) \cdot\left(\vec{r}_{2}-\vec{r}_{1}\right)$, and from this it can be shown that

$$
\dot{s}=\frac{v_{1}\left(r_{\alpha} u+\sin (\alpha)\right)}{\sin (\alpha-\phi)} .
$$

and

$$
\dot{r}_{\alpha}=v_{1} \frac{\sin (\phi)+r_{\alpha} u \cos (\alpha-\phi)}{\sin (\alpha-\phi)} .
$$

Now let us find the equation for $\dot{\phi}$. From Fig. 2, we can see that the angle between $\vec{x}_{1}$ and $\vec{y}_{2}$ is $\left(\frac{\pi}{2}-\phi\right)$. Therefore, we get

$$
\sin (\phi)=\vec{x}_{1} \cdot \vec{y}_{2},
$$

from which it follows that the equation for $\dot{\phi}$ is

$$
\dot{\phi}=v_{1} r_{\alpha} u\left(\frac{1}{r_{\alpha}}-\frac{\kappa}{\sin (\alpha-\phi)}\right)-\frac{v_{1} \sin (\alpha) \kappa}{\sin (\alpha-\phi)} .
$$

For the Sting-I autonomous vehicle, the sensor on each side of the vehicle is installed such that $\alpha=\frac{\pi}{2}$. In this case, (10) is simplified as

$$
\dot{s}=\frac{v_{1}\left(r_{\alpha} u+1\right)}{\cos (\phi)} .
$$

(11) is simplified as

$$
\dot{r}_{\alpha}=v_{1} \tan (\phi)\left(1+r_{\alpha} u\right) .
$$

Also, (13) is simplified as

$$
\dot{\phi}=v_{1} u\left(1-\frac{r_{\alpha} \kappa}{\cos (\phi)}\right)-\frac{\kappa v_{1}}{\cos (\phi)} .
$$

The system equations are different from the equations for the closest point in [2].

\section{Controller Design And Convergence ANALYSIS}

\section{A. Lyapunov function}

Consider the Lyapunov function candidate

$$
V_{1}=-\ln (\cos (\phi))+h\left(r_{\alpha}\right),
$$

$h\left(r_{\alpha}\right)$ in (17) should satisfy these conditions:

1) $d h / d r_{\alpha}=f\left(r_{\alpha}\right)$,where $f\left(r_{\alpha}\right)$ is a Lipschitz continuous function on $(0, \infty)$, so that $h\left(r_{\alpha}\right)$ is continuously differentiable on $(0, \infty)$.

2) $\lim _{r_{\alpha} \rightarrow 0} f\left(r_{\alpha}\right)=-\infty$, which leads to $\lim _{r_{\alpha} \rightarrow 0} h\left(r_{\alpha}\right)=$ $\infty$. This is needed to blow up $V_{1}$ as the moving vehicle approaches collision with the boundary curve.

3) $f\left(r_{\alpha}\right)$ vanishes at a point where $r_{\alpha}=r_{0}$. At which point, $h\left(r_{\alpha}\right)$ is also zero. This is for the moving vehicle to converge to the desired relative position at a distance from the boundary curve given by $r_{\alpha}=r_{0}$.
4) $\lim _{r \rightarrow \infty} h\left(r_{\alpha}\right)=\infty$. By this condition and the form of $V_{1}^{\infty}$, we conclude that $V_{1}$ is radially unbounded (i. e., $V_{1} \rightarrow \infty$ as $\|\phi\| \rightarrow \pi / 2$, as $r_{\alpha} \rightarrow 0$, or as $\left.r_{\alpha} \rightarrow \infty\right)$.

Observe that $V_{1}$ given by (17) is continuously differentiable provided (3) holds. In (17), the term $-\ln (\cos (\phi))$ penalizes misalignment between the tangent vector of the moving vehicle with the tangent vector to the boundary curve at the detected point. The term $h\left(r_{\alpha}\right)$ in (17) deals with the separation between the moving vehicle and the boundary curve. In short, $V_{1}$ is designed to make a vehicle converge to the relative position where $r_{\alpha}=r_{0}$ and $\phi=0$. Here, $r_{0}$ represents the desired separation between the moving vehicle and the boundary curve for boundary-following. This form of Lyapunov function has been used in recent papers regarding boundary following and curve tracking using the closest point information in [2], and [6].

For the point detected by the fixed range sensors at an angle $\alpha=\pi / 2$, our candidate $f\left(r_{\alpha}\right)$ satisfying these conditions is

$$
f\left(r_{\alpha}\right)=\frac{-1}{r_{\alpha}}+\frac{1}{r_{0}}
$$

where $r_{0}$ is a positive constant which represents the desired separation between the moving vehicle and the boundary curve for boundary-following. Further, the corresponding $h\left(r_{\alpha}\right)$ is

$$
h\left(r_{\alpha}\right)=-\ln \left(r_{\alpha}\right)+\frac{r_{\alpha}}{r_{0}}+\ln \left(r_{0}\right)-1,
$$

which satisfies the conditions for $h\left(r_{\alpha}\right)$ in $V_{1}$ (17).

The time derivative of $V_{1}$ is

$$
\begin{array}{r}
\dot{V}_{1}=-\tan (\phi)\left[u\left(\frac{v_{1} r_{\alpha} \kappa}{\cos (\phi)}-v_{1}-v_{1} f\left(r_{\alpha}\right) r_{\alpha}\right)\right. \\
\left.+\frac{v_{1}}{\cos (\phi)} \kappa-v_{1} f\left(r_{\alpha}\right)\right],
\end{array}
$$

where we have used (15), (16), and (17). We now assume that the speed $v_{1}>0$ is a constant and design steering control $u$ so that $\dot{V}_{1} \leq 0$.

\section{B. Tracking control for convex curves}

We first consider the case when the curve is convex and curving away from the vehicle. In this case we have $\kappa<0$.

One choice of $u$ which leads to $\dot{V}_{1} \leq 0$ is

$$
u_{1}=\frac{v_{1} \kappa-\cos (\phi)\left(v_{1} f\left(r_{\alpha}\right)+\mu \sin (\phi)\right)}{v_{1}\left(\cos (\phi)+f\left(r_{\alpha}\right) r_{\alpha} \cos (\phi)-r_{\alpha} \kappa\right)},
$$

where $\mu>0$ is a constant. The time derivative of $V_{1}$ in (20) with $u$ given by (21) is

$$
\dot{V}_{1}=-\mu \frac{\sin ^{2}(\phi)}{\cos (\phi)} \leq 0
$$


where (3) is used. Thus, $\dot{V}_{1} \leq 0$ and $\dot{V}_{1}=0$ if and only if $\sin (\phi)=0$. But by (3), we see that $\dot{V}_{1}=0$ if and only if $\phi=0$.

From now on, we define the case where the denominator of control law is zero as singular case of the controller. It seems possible that the control law given by (21) is singular when the denominator of $u_{1}$ equals to zero, i.e., $\cos (\phi)=\frac{r_{\alpha} \kappa}{1+f\left(r_{\alpha}\right) r_{\alpha}}$. Using (18), we have

$$
\cos (\phi)=\frac{r_{\alpha} \kappa}{1+f\left(r_{\alpha}\right) r_{\alpha}}=r_{0} \kappa
$$

Therefore, in the case where the curvature of the lane at the detected point $\kappa$ is equal to or smaller than zero in (23), the denominator of the control law in (21) will never be zero since $\cos (\phi)>0$, and we can now state the following theorem:

Theorem 1: Consider the case where the boundary curve is convex, i.e., $\kappa<0$. Then, using the steering control law in (21), the vehicle satisfying (3) with constant speed $v_{1}>0$ tracks the curve at a distance $r_{0}$ without collision.

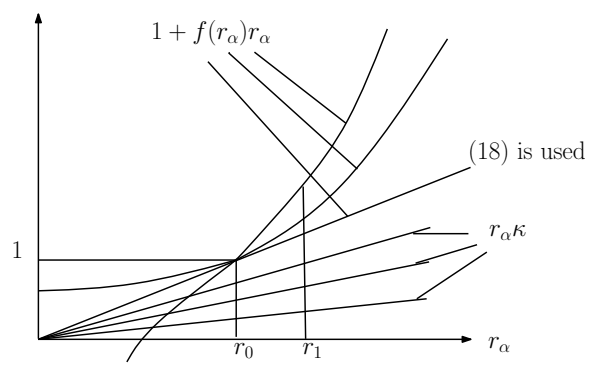

Fig. 3. Comparison of $1+f\left(r_{\alpha}\right) r_{\alpha}$ and $\kappa r_{\alpha}$. Control law given by (21) is singular when $\cos (\phi)=\frac{r_{\alpha} \kappa}{1+f\left(r_{\alpha}\right) r_{\alpha}}$. We argue that singular case can not be removed by choosing $f\left(r_{\alpha}\right)$ if the curvature $\kappa$ is upper bounded.

\section{Control laws for concave curve with bounded curva- ture}

We consider the case when the curve is concave, i.e. curving towards the vehicle. In this case we have $\kappa>0$. It is possible that the control law given by (21) is singular when the denominator of $u_{1}$ equals to zero, i.e., $\cos (\phi)=$ $\frac{r_{\alpha} \kappa}{1+f\left(r_{\alpha}\right) r_{\alpha}}$. However, in the case where the curvature of the lane at the detected point $\kappa$ is bigger than $\frac{1}{r_{0}}$ in (23), no uncontrollable case happens because $|\cos \phi| \leq 1$.

In the real experimental environment, it is necessary for the vehicle to follow a boundary curve whose curvature is sufficiently small, such as a straight line. We argue that in this case the singular case exists regardless of the choice of $f\left(r_{\alpha}\right)$.

Fig. 3 shows possible graphs of $1+f\left(r_{\alpha}\right) r_{\alpha}$ and $r_{\alpha} \kappa$ respectively. When (18) is used as $f\left(r_{\alpha}\right)$, we get $1+$ $f\left(r_{\alpha}\right) r_{\alpha}=\frac{r_{\alpha}}{r_{0}}$. Therefore, the straight line connecting the origin and $\left(r_{0}, 1\right)$ represents $1+f\left(r_{\alpha}\right) r_{\alpha}$ when (18) is used as $f\left(r_{\alpha}\right)$. In Fig.3, regardless of $f\left(r_{\alpha}\right), 1+f\left(r_{\alpha}\right) r_{\alpha}$ is a continuous function which is equal to 1 when $r_{\alpha}=$ $r_{0}$. Also, regardless of the decreasing rate of $f\left(r_{\alpha}\right)$ as $r_{\alpha} \downarrow 0$, we can assure that $\lim _{r_{\alpha} \downarrow 0} 1+f\left(r_{\alpha}\right) r_{\alpha} \leq 1$. As $r_{\alpha} \downarrow r_{0}$, we see that $f\left(r_{\alpha}\right)$ and $r_{\alpha}$ both decrease to make $\left(1+f\left(r_{\alpha}\right) r_{\alpha}\right)$ decrease for any choice of $f\left(r_{\alpha}\right)$.

At the same time, the possible $r_{\alpha} \kappa$ are plotted as the straight lines. If the curvature $\kappa$ is upper bounded by $\frac{1}{r_{0}}$, then these straight lines will be below the curve that represents $\left(1+f\left(r_{\alpha}\right) r_{\alpha}\right)$, regardless of what $f\left(r_{\alpha}\right)$ is. Therefore, $\frac{r_{\alpha} \kappa}{1+f\left(r_{\alpha}\right) r_{\alpha}}<1$ and $\cos \phi=\frac{r_{\alpha} \kappa}{1+f\left(r_{\alpha}\right) r_{\alpha}}$ always has a solution for $\phi$. This singular case can not be removed by choosing $f\left(r_{\alpha}\right)$.

\section{The safety zone}

As seen on the form of (23), singular case will never happen if $|\phi|<\arccos \left(r_{0} \kappa_{M}\right)$, where $\kappa_{M}$ is the upper bound of $\kappa$. Thus, we define the set $U=$ $\left\{\left(r_{\alpha}, \phi\right) \mid V_{1}\left(r_{\alpha}, \phi\right)<-\ln \left(\left|r_{0} \kappa_{M}\right|\right)\right\}$ as the safety zone. The controller (21) is used inside the safety zone. Since this controller yields $\dot{V}_{1} \leq 0$, we conclude that once the vehicle under control enters the safety zone $U$, it will never leave. Therefore, according to Theorem 1, the curve tracking behavior is stabilized without collision.

\section{E. Switching control that aims for the safety zone}

When the vehicle is initially out of the safety zone but, during its movements, it will come close to the set where $\cos (\phi)=r_{0} \kappa$, control law (21) can not be applied due to singularity.

We develop a switching system as depicted in Fig.4 to steer the system into the safety zone in finite time. Four cases are distinguished, which correspond to four sets $G_{1}, G_{2}, G_{3}$ and $G_{4}$ defined as follows:

$$
\begin{aligned}
& G_{1}=\left\{\left(r_{\alpha}, \phi\right) \mid\left\|\cos (\phi)-r_{0} \kappa\right\|>\epsilon \text { but }\left(r_{\alpha}, \phi\right) \notin U\right\} \\
& G_{2}=\left\{\left(r_{\alpha}, \phi\right) \mid \epsilon_{2}<\left\|\cos (\phi)-r_{0} \kappa\right\| \leq \epsilon\right\} \\
& G_{3}=\left\{\left(r_{\alpha}, \phi\right) \mid\left\|\cos (\phi)-r_{0} \kappa\right\| \leq \epsilon_{2}\right\} \\
& G_{4}=U,
\end{aligned}
$$

where $\epsilon_{2}<\epsilon$.

Three control laws are designed for these four cases. When the system states are in $G_{1}$ or $G_{4}$, we use $u_{1}$ in (21). When the states enter $G_{2}$ from $G_{1}$, we switch to $u_{2}$ which is

$$
u_{2}=\frac{v_{1} \kappa-\cos (\phi)\left(v_{1} f\left(r_{\alpha}\right)+\mu_{2} \sin (\phi)\right)}{v_{1}\left(\cos (\phi)+f\left(r_{\alpha}\right) r_{\alpha} \cos (\phi)-r_{\alpha} \kappa\right)},
$$




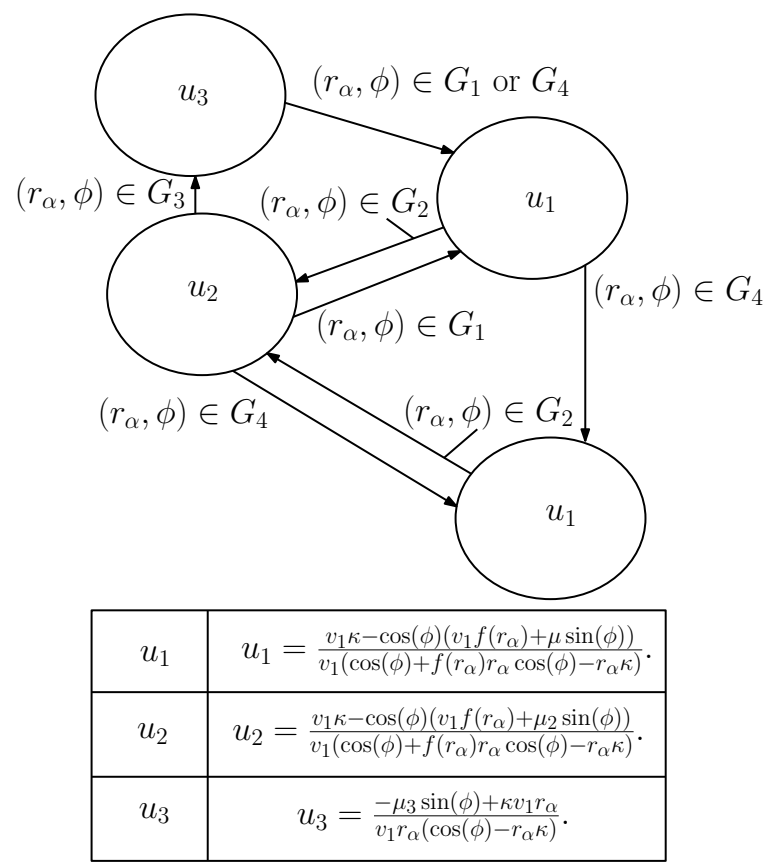

Fig. 4. The switching control strategy used to enter safety zone. $u_{1}$ in (21) is used in normal situations i.e. when the states are in $G_{1}$ or $G_{4}$. We switch to $u_{2}$ in (25) when the states enter $G_{2}$ and switch to $u_{3}$ in (27) when the states enter $G_{3}$.

where the only difference between $u_{1}$ and $u_{2}$ is that the gain $\mu_{2}$ is much bigger than $\mu$. The time derivative of $V_{1}$ under control $u_{2}$ given by (25) is

$$
\dot{V}_{1}=-\mu_{2} \frac{\sin ^{2}(\phi)}{\cos (\phi)} \leq 0 .
$$

When the states of the system enter $G_{3}$ from $G_{2}$ $\left(r_{\alpha}, \phi\right) \in G_{3}$, we switch to controller $u_{3}$ :

$$
u_{3}=\frac{-\mu_{3} \sin (\phi)+\kappa v_{1} r_{\alpha}}{v_{1} r_{\alpha}\left(\cos (\phi)-r_{\alpha} \kappa\right)},
$$

where $\mu_{3}>0$ is a constant. Under this controller, we have

$$
\dot{\phi}=-\frac{\mu_{3} \tan (\phi)}{r_{\alpha}} .
$$

Hence, $\phi \rightarrow 0$ as $t \rightarrow \infty$. This implies that the system states will get out of $G_{3}$ and then out of $G_{2}$ in finite time. We switch back to controller $u_{1}$ after the states enter either $G_{1}$ or $G_{4}$. Note that by Theorem 1 , once the states enter $G_{4}$, they will stay in $G_{4}$ and converge to the desired values.

We now prove convergence of the system under the switching control laws illustrated in Fig. 4. The idea is that the value of the Lyapunov function $V_{1}$ may be increasing under controller $u_{3}$, but such increase will be compensated by controller $u_{2}$. Hence the overall effect is that the Lyapunov function decreases until the system reaches $G_{4}$. Some notations and technical conditions are needed to rigorously state and prove the results.

It is uninteresting if the states never enters the set $G_{3}$. In which case $V_{1}$ would be decreasing until $G_{4}$ is reached. Therefore, we discuss the most general case i.e. the states of the system enters $G_{3}$ for a number of times. In order to enter $G_{3}$, the system must enter $G_{2}$ first. We use the notations $t_{1}^{i}$ to indicate the time when the system enters $G_{2}, t_{2}^{i}$ to indicate the time when the system enters $G_{3}$, and $t_{3}^{i}$ to indicate the time when the system leaves $G_{2}$. The index $i$ is used to distinguish multiple entries. If the states enter $G_{3}$ and later leaves $G_{2}$, then $t_{1}^{i}, t_{2}^{i}$ and $t_{3}^{i}$ happen in sequence.

The following technical assumptions are needed

(A1) The curvature $\kappa$ is bounded above by $\kappa_{M}>0$.

(A2) The desired distance $r_{0}$ satisfies that $r_{0} \kappa_{M}<1$.

(A3) Define $\zeta$ as

$v_{1}\left\|-\arccos \left(\kappa_{M} r_{0}+\epsilon\right)+\arccos \left(\kappa_{M} r_{0}-\epsilon_{2}\right)\right\|+\epsilon_{3}$, where $\epsilon_{3}>0$ is a constant. We assume that the gains $\mu_{2}$ and $\mu_{3}$ in controllers $u_{2}$ and $u_{3}$ satisfy $\mu_{2} \mu_{3}\left(t_{2}^{i}-t_{1}^{i}\right)>\frac{\zeta r_{0} \kappa_{M}}{1-\left(r_{0} \kappa_{M}\right)^{2}}$ for all $i$.

Assumptions (A1) and (A2) put mild constraints on the curve to follow. Assumption (A3) is the key technical assumption. This assumption can always be satisfied when $t_{2}^{i}-t_{1}^{i} \neq 0$ and if we allow the gains $\mu_{2}$ or $\mu_{3}$ to be arbitrarily large.

Theorem 2: Consider the system defined by (15) and (16) governing the relative distance and heading angle between the vehicle and the detected point. Suppose the vehicle travels at constant speed $v_{1}$. Under the switching strategy in Fig. 4, with assumptions (A1)-(A3) satisfied, the states of the switching closed loop system enter $G_{4}$ in finite time.

We omit the proof of this theorem, but note that it should be organized along two steps, namely

1) Show that when $u_{3}$ is used, $V_{1}$ will increase a finite amount bounded above in the worst case.

2) Show that when $u_{2}$ is used, $V_{1}$ will decrease more than the upper bound for its increase under $u_{3}$.

In Fig.5, a typical switching process is plotted. Controller $u_{1}$ is used from 0 to $t_{1}^{i}, u_{2}$ is used from $t_{1}^{i}$ to $t_{2}^{i}, u_{3}$ is used from $t_{2}^{i}$ to $t_{3}^{i}$, and $u_{1}$ is used again after $t_{3}^{i}$. Intervals of using $u_{2}$ is long enough to overcome the increase of $V_{1}$ inside the interval when $u_{3}$ is used. $V_{1}$ always decrease more than it increases.

In the case where $r_{\alpha}=r_{0}$ and $\cos (\phi)=r_{0} \kappa$, we have singular cases of $u_{1}, u_{2}$ and $u_{3}$ at the same time. This singular case will not happen if the vehicle is in the safety zone. An example of applying the switched 


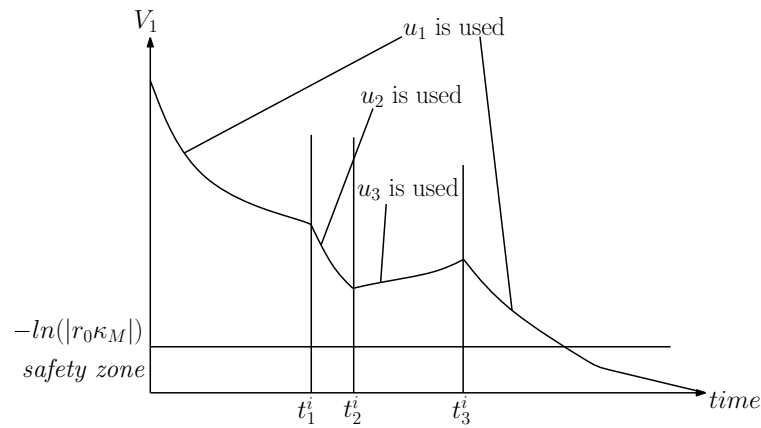

Fig. 5. The Lyapunov function $V_{1}$ in a typical case of switching control. $u_{1}$ in (21) is used from 0 to $t_{1}^{i}, u_{2}$ in (25) is used from $t_{1}^{i}$ to $t_{2}^{i}, u_{3}$ in (27) is used from $t_{2}^{i}$ to $t_{3}^{i}$, and $u_{1}$ is used from $t_{3}^{i}$ to final time.

control strategy is shown in Fig 6, in which switches are needed to overcome the singular case.

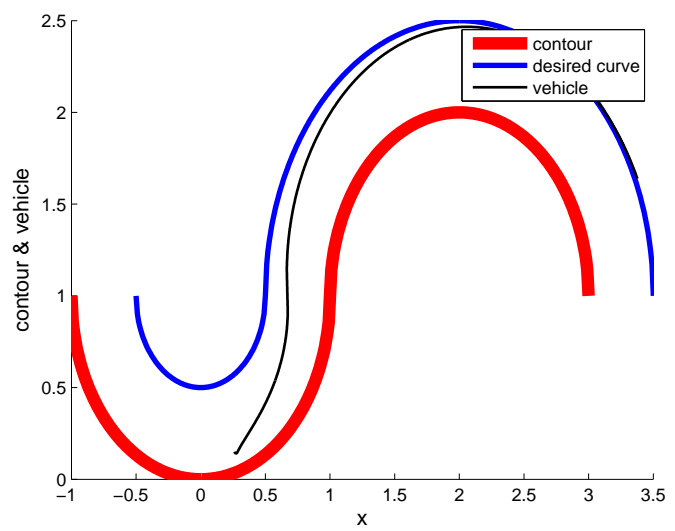

Fig. 6. The vehicle is close to the set where $\cos (\phi)=\kappa r_{0}$ initially. However, switched control strategy is used to overcome the singular case.

\section{REFERENCES}

[1] M. Egerstedt, X. Hu, and A. Stotsky, "Control of mobile platforms using a virtual vehicle approach," vol. 46, pp. 1777-1782, November 2001.

[2] F. Zhang, E. Justh, and P. S. Krishnaprasad, "Boundary following using gyroscopic control," in Proc. of 43rd IEEE Conf. on Decision and Control. Atlantis, Paradise Island, Bahamas: IEEE, 2004, pp. 5204-5209.

[3] F. Zhang, A. O'Connor, D. Luebke, and P. S. Krishnaprasad, "Experimental study of curvature-based control laws for obstacle avoidance," in Proceedings of 2004 IEEE International Conf. on Robotics and Automation. New Orleans, LA: IEEE, 2004, pp. 3849-3854.

[4] F. Zhang and N. E. Leonard, "Coordinated patterns of unit speed particles on a closed curve," Systems and Control Letters, vol. 56, no. 6, pp. 397-407, 2007.

[5] F. Zhang, E. Fiorelli, and N. E. Leonard, "Exploring scalar fields using multiple sensor platforms: Tracking level curves," in Proc. of 46th IEEE Conf. on Decision and Control, New Orleans, LA, 2007, pp. 3579-3584.
[6] F. Zhang, D. M. Fratantoni, D. Paley, J. Lund, and N. E. Leonard, "Control of coordinated patterns for ocean sampling," International Journal of Control, vol. 80, no. 7, pp. 1186-1199, 2007.

[7] C. Samson, "Control of chained systems: Application to pathfollowing and time-varying point-stabilization of mobile robots," IEEE Trans. on Automatic Control, vol. 40, no. 1, pp. 64-77, 1995.

[8] R. Frezza and G. Picci, "On line path following by recursive spline updating," in Proc. of the 34th Conf. on Decision and Control, ser. 13-15, vol. 4, New Orleans, December 1995, pp. $4047-4052$.

[9] Y. Ma, J. Koseck'a, and S. Sastry, "Vision guided navigation for a nonholonomic mobile robot," in Proc. of the 36th IEEE Conference on Decision and Control, ser. 10-12, vol. 3, December 1997 , pp. $3069-3074$.

[10] E. D. Dickmanns and V. Graefe, "Applications of dynamic monocular machine vision," Machine Vision and Applications, vol. 1, no. 4, pp. 241-261, 1988.

[11] E. D. Dickmanns and B. D. Mysliwetz, "Recursive 3-d road and relative ego-state estimation," IEEE Transactions on Pattern Analysis and Machine Intelligence, vol. 14, no. 2, pp. 199-213, 1992.

[12] D. Raviv and M. Herman, "A nonreconstruction approach for road following," Proc. of SPIE: Intelligent Robots and Computer Vision, vol. 1608, no. 5, pp. 2-12, 1992.

[13] E. W. Justh and P. S. Krishnaprasad, "Steering laws for motion camouflage," Royal Society of London Proceedings Series A, vol. 462, pp. 3629-3643, Dec. 2006.

[14] K.Li and J.Baillieul, "Data-rate requirements for nonlinear feedback control," in Proc. 6th IFAC Symp. Nonlinear Contr. Sys., Uni.Stuttgart, Germany, 2004, pp. 1277-1282.

[15] A. Micaelli and C. Samson, "Trajectory tracking for unicycletype and two-steering-wheels mobile robots," INRIA, Tech. Rep. RR-2097, November 1993.

[16] S. B. Andersson and J. Park, "Tip steering for fast imaging in AFM," in Proc. 2005 American Control Conf., Portland, OR, June 6-10, 2005, pp. 2469-2474.

[17] J. Clark and R. Fierro, "Cooperative hybrid control of robotic sensors for perimeter detection and tracking." 2005 American Control Conference, 2005, pp. 3500 - 3505.

[18] D. Baronov and J. Baillieul, "Reactive exploration through following isolines in a potential field," in American Control Conference, July 2007, pp. 2141 - 2146.

[19] J.P.Hespanha and A.S.Morse, "Stability of switched systems with average dwell-time," in Proceedings 38th Conference on Decision and Control, vol. 3, 1999, pp. 2655 - 2660.

[20] D. Liberzon and A. S. Morse, "Benchmark problems in stability and design of switched systems," IEEE Control Systems Magazine, Tech. Rep., October 1999.

[21] M. Branicky, "Multiple lyapunov functions and other analysis tools for switched and hybrid systems," in IEEE Trans. Automatic Control, vol. 43(4), April 1998, pp. 475-482.

[22] S. P. R. DeCarlo, M.S. Branicky and B. Lennartson, "Perspectives and results on the stability and stabilizability of hybrid systems," in Proceedings of the IEEE, vol. 88(2), July 2000, pp. 10691082.

[23] T. Hu, "Switching law construction for discrete-time systems via composite quadratic functions." 2007 American Control Conference, 2007, pp. $675-680$.

[24] C. Tomlin and S. Sastry, "Switching through singularities," in Proceedings of the 36th IEEE Conference on Decision and Control, vol. 1, December 1997, pp. 1-6.

[25] M. D. Carmo, Differential Geometry of Curves and Surfaces. Prentice Hall, 1976. 